\title{
Erratum: Coalescence of viscous two-dimensional smectic islands [Phys. Rev. E 99, 062702 (2019)]
}

N. S. Shuravin, P. V. Dolganov, and V. K. Dolganov

(Q) (Received 21 June 2019; published 17 July 2019)

DOI: 10.1103/PhysRevE.100.019901

This paper contains misprints. On p. 3, in the line following Eq. (2), in group $\frac{\eta R}{\gamma}$, a factor of $\sqrt{2}$ was lost. Group $\eta R / \gamma$ should read as $\sqrt{2} \eta R / \gamma$. On p. 4, left column, a factor of $\sqrt{2}$ was lost, time $\tau_{R}$ in the seventh line of the second paragraph should read as $\tau_{R}=\sqrt{2} \eta R / \gamma$. On p. 4, the caption for Fig. 5 and left column, in the ninth line of the second paragraph, time $\tau_{R T}^{*}$ should read as $\tau_{R T}^{*}=\sqrt{2} b \eta R / N \gamma_{d}$. In the caption for Fig. $6, H(t) / R$ should read as $H(t) R=-2\left(t / \pi \tau_{R}\right) \ln \left(t / \tau_{R}\right)$. These changes have no effect on the rest of the paper. The figures, results, discussion, and conclusions remain valid. 